notent une diminution rapide du phosphore lipidique des globules de matière grasse associée à une montée brusque de la mobilité électrophorétique à environ 60 à $65 \%$ de matière grasse. D'après MoHr et Weldm $\left(1948\right.$ b), la viscosité de la crème mesurée à $40^{\circ}$ et à $60^{\circ}$ augmente rapidement à $65-70 \%$ de matière grasse. Cette usure de la substance de la membrane peut n'être que partielle, puisque la crème à $80 \%$ conserve son caractère d'émulsion graissedans-eau au-dessus de $20^{\circ}$.

Le lait écrémé provenant du concentrateur contient plus de matière grasse que le lait écrémé primitif $(0,04$ à $0,05 \%$ par extraction à l'éther); mais moins de matière grasse que le babeurre ordinaire $(0,5$ à $0,9 \%)$. D'après MuLDER et ses collègues (1949), la teneur en matière grasse du second lait écrémé est habituellement inférieure à $0,07 \%$ (par la méthode Gerber), ne s'élevant à $0,1 \%$ que dans quelques cas. LoFtus-HILls, BALLARD et Wilkinson (1950) indiquent $0,1 \%$ (par extraction à l'éther) comme étant la proportion la plus élevée pour le lait écrémé provenant de crème pasteurisée dans un appareil à plaques. McDowall et McWhirTer (1948) ont, toutefois, trouvé des proportions aussi élevées que $0,6 \%$ pour le lait pasteurisé provenant de crème pasteurisée instantanément.

La teneur en matière grasse du second lait écrémé est influencée par le pré-traitement de la crème. Elle est augmentée par l'agitation. pandant le transport et par la vacréation de la crème primitive (McDowall et MoWhirter, 1948 ; Loftus Hills et Coll., 1950), une légère vacréation l'augmentant jusqu'à $0,38 \%$ et une vacréation poussée jusqu'à $0,49 \%$.

L'utilisation du second lait écrémé acidifié comme succédané du babeurre a été étudié par Moнr et HäsING (1949) et par MонR. et MACK (1944).

(A suivre)

\title{
REVUE
}

\section{PRODUCTION DE LAIT ET DÉRIVÉS EN EUROPE ET DANS LE MONDE (1)}

La production de lait de vache, qui était en 1949 sensiblement voisine de celle d'avant-guerre, a dépassé celle-ci en 1950, et a marqué une autre augmentation en 1951.

Nous indiquons ci-dessous les données de la production mondiale de lait de vache :

(1) Extrait de $1 l$ Mando del Latte, 1952, 7, 391. 


\begin{tabular}{|c|c|c|c|}
\hline \multirow[t]{2}{*}{ Pays } & \multicolumn{3}{|c|}{$\begin{array}{c}\text { Production de lait de vache } \\
\text { en milliers de tonnes }\end{array}$} \\
\hline & $1937-1939$ & I 949 & 1950 \\
\hline Europe $\ldots \ldots \ldots \ldots \ldots \ldots \ldots \ldots$ & 96.300 & 80.000 & 87.000 \\
\hline Amérique du Nord et du Centre ... & 57.100 & 65.600 & 66.100 \\
\hline Amérique du Sud $\ldots \ldots \ldots \ldots \ldots$ & 6.600 & 8.700 & 8.600 \\
\hline Asie $\ldots \ldots \ldots \ldots \ldots \ldots \ldots$ & 9.600 & 10.700 & i1. 300 \\
\hline Afrique $\ldots \ldots \ldots \ldots \ldots \ldots$ & 4.000 & 5.200 & 5.400 \\
\hline \multirow[t]{2}{*}{ Océanie $\ldots \ldots \ldots \ldots \ldots \ldots$} & 9.800 & 10.300 & 10.600 \\
\hline & 183.400 & 180.500 & 189.000 \\
\hline
\end{tabular}

La production de lait de brebis, de chèvre et de buffle qui, selon les assertions les plus récentes, s'élevait à 14,5 millions de tonnes avant la guerre, a atteint en 1949 le chiffre de 15,2 millions de tonnes, et en 1950 , la quantité totale de 15 millions et demi de tonnes.

Les augmentations de la production de lait de vache par rapport à la période d'avant-guerre, se sont avérées correspondre aux pourcentages suivants : Afrique, $35 \%$; Amérique du Sud, $30 \%$; Asie, $17 \%$; Amérique du Nord et du Sud, $16 \%$; Océanie, $8 \%$.

Les pays européens accusent une production inférieure de $10 \%$ à celle d'avant-guerre, mais on a des raisons de croire que cette différence s'est annulée au cours de 1951.

Dans notre continent, la production de lait de vache d'avantguerre a été dépassée dans les pays suivants : Belgique, Danemark, France, Irlande, Pays-Bas, Norvège, Suède, Royaume-Uni.

La Finlande, la Suisse et 1'Italie se sont rapprochées en 1950 du chiffre de production d'avant-guerre, comme le montre le tableau suivant :

\begin{tabular}{|c|c|c|c|}
\hline \multirow{2}{*}{ Pays } & \multicolumn{3}{|c|}{$\begin{array}{l}\text { Production de lait de vache } \\
\text { en milliers de tonnes }\end{array}$} \\
\hline & $1937-1939$ & 1949 & 1950 \\
\hline Autriche... & 1.980 & 1.740 & 1.890 \\
\hline Belgique $\ldots \ldots \ldots \ldots \ldots \ldots \ldots$ & 2.830 & 3.060 & 3.230 \\
\hline Tehécoslovaquie ............. & 4.430 & 2.600 & 2.600 \\
\hline 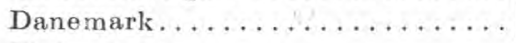 & 5.270 & 4.880 & 5.400 \\
\hline 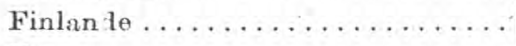 & 2.580 & 2.170 & 2.450 \\
\hline Frane $\ldots \ldots \ldots \ldots \ldots \ldots \ldots$ & 14.190 & 13.250 & 15.000 \\
\hline
\end{tabular}




\begin{tabular}{|c|c|c|c|}
\hline \multirow[t]{2}{*}{ Pays } & \multicolumn{3}{|c|}{$\begin{array}{l}\text { Production de lait de vache } \\
\text { en milliers de tonnes }\end{array}$} \\
\hline & 1950 & 1949 & 1937.39 \\
\hline Sarre $\ldots \ldots \ldots \ldots \ldots \ldots$ & 80 & 50 & 60 \\
\hline Allemagne Occidentale ......... & 15.020 & 11.880 & 13.950 \\
\hline Allemagne Orientale .......... & 4.940 & 2.350 & 2.350 \\
\hline Grèce $\ldots \ldots \ldots \ldots \ldots \ldots \ldots$ & 140 & 90 & 110 \\
\hline Hongrie $\ldots \ldots \ldots \ldots \ldots \ldots \ldots$ & 1.530 & 740 & 740 \\
\hline Irlande $\ldots \ldots \ldots \ldots \ldots \ldots$ & 2.300 & 2.300 & 2.400 \\
\hline Islande $\ldots \ldots \ldots \ldots \ldots$ & 60 & 70 & 70 \\
\hline Italie $\ldots \ldots \ldots \ldots \ldots \ldots$ & 5.920 & 5.170 & 5.450 \\
\hline Yougoslavie ............... & 2.740 & 2.350 & 2.450 \\
\hline Luxembourg................. & 140 & 160 & 160 \\
\hline Pays-Bas ................ & 4.940 & 5.460 & 5.770 \\
\hline Norvège ..................... & 1.330 & 1.520 & 1.610 \\
\hline Pologne ................. & 10.300 & 3.390 & 3.390 \\
\hline Suède. . . . . . . . . . . . . & 4.590 & 4.640 & 4.890 \\
\hline Suisse $\ldots \ldots \ldots \ldots \ldots \ldots \ldots$ & 2.650 & 2.440 & 2.570 \\
\hline \multirow[t]{2}{*}{ 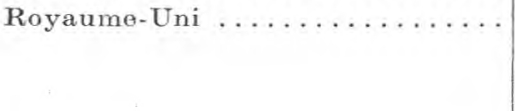 } & 8.340 & 9.780 & 10.520 \\
\hline & 96.300 & 80.090 & 87.060 \\
\hline
\end{tabular}

La production de beurre mondiale est exprimée comme suit :

\begin{tabular}{|c|c|c|c|}
\hline Europe ................ & 1.500 & 1.300 & $: \quad 1.460$ \\
\hline Amérique du Nord et du Centre .. & 1.100 & 900 & 900 \\
\hline Amérique du sud $\ldots \ldots \ldots \ldots$ & 60 & 70 & 80 \\
\hline 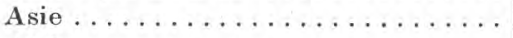 & 70 & 70 & 70 \\
\hline Afrique $\ldots \ldots \ldots \ldots \ldots \ldots$ & 30 & 40 & 50 \\
\hline \multirow[t]{2}{*}{ Océanie } & 380 & 340 & 350 \\
\hline & 3.140 & 2.720 & 2.910 \\
\hline
\end{tabular}

La production spécifique des Pays européens a été la suivante (voir tableau ci-après).

A l'examen des données ci-après exposées, il ressort que la production beurrière mondiale est partout inférieure de $10 \%$ à celle d'avant-guerre, que l'Europe a presque atteint le volume de cette production d'avant-guerre, que l'on enregistre une augmentation dans les Pays de l'Amérique du Sud et de l'Afrique du Sud, et que la régression la plus sensible se rencontre en Amérique du Nord (18\% en moins par rapport à avant la guerre; presque totalement aux U.S.A. et, pour de petites quantités, au Canada), 


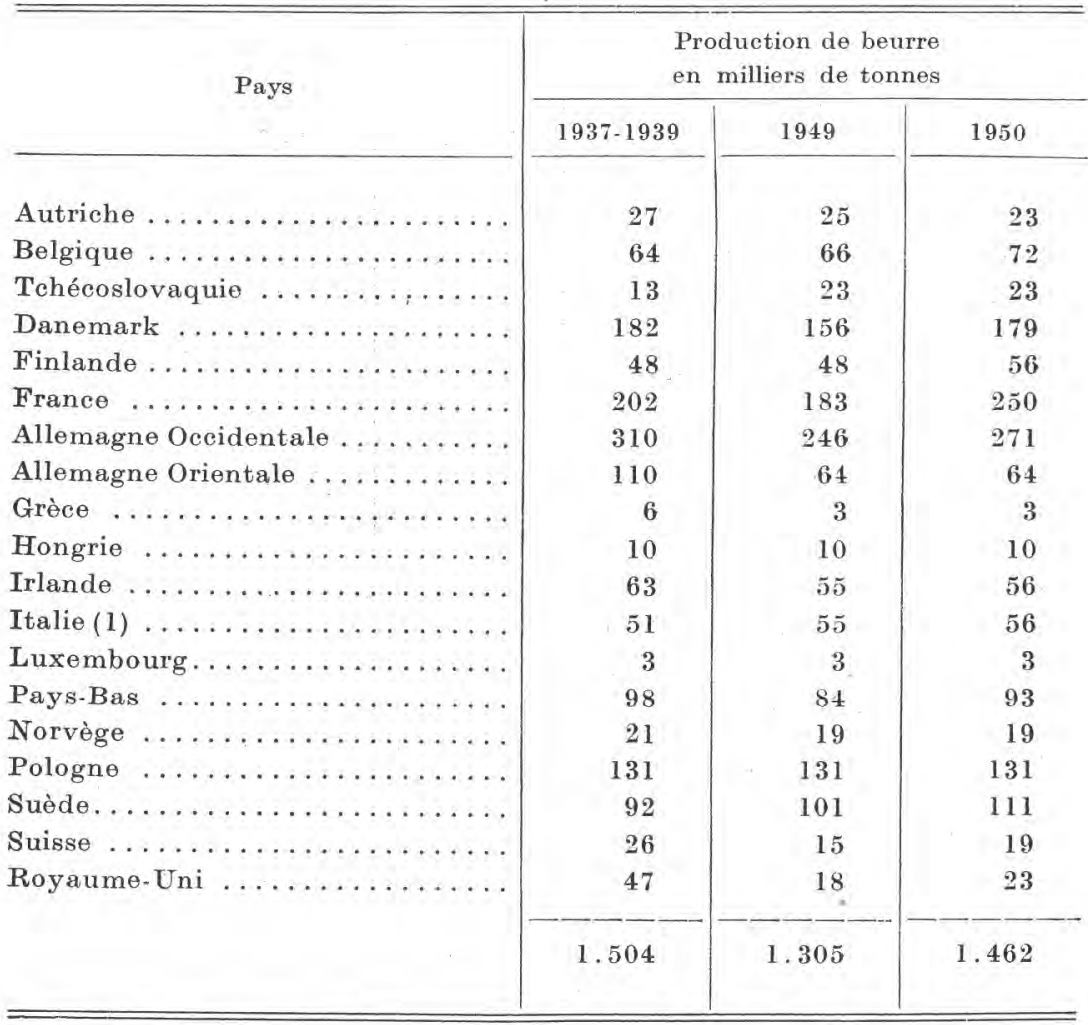

et en Océanie ( $8 \%$ en moins en Australie, avec une légère augmentation en Nouvelle-Zélande).

Dans les pays européens, et toujours par rapport à la période d'avant-guerre, la régression la plus sensible se constate en Irlande, en Grèce, en Suisse et au Royaume-Uni, alors que la production beurrière augmente en Belgique, en Finlande, en France, en Italie et en Suède.

Le rapport entre la production mondiale de fromage des trois années 1937-1939 et celle de 1949 et de 1950 apparaît d'après les chiffres suivants (voir tableau ci-nprès.

La production de fromage, qui avait dépassé de $6 \%$ en 1948 celle d'avant-guerre, a été augmentée de $22 \%$ en 1950, avec un accroissement mondial de 387.000 tonnes.

Cette augmentation a été de l'ordre de : 261.000 tonnes en

(1) Dans la statistique publiée par la Revue (n 8 de 1951), la production italienne de la période d'après-guerre était évaluée à 50.000 tonnes, alors que selon les relevés de "l'Assolatten (Association du Lait), elle atteint à peine 42.000 tonnes. Les relevés de "l'Assolatten indiquent 48.000 tonnes pour 1949 et 53.000 tonnes pour 1950. 


\begin{tabular}{|c|c|c|c|}
\hline \multirow[t]{2}{*}{ Pays } & \multicolumn{3}{|c|}{$\begin{array}{l}\text { Production de fromage } \\
\text { en milliers de tonnes }\end{array}$} \\
\hline & 1937.1939 & 1949 & 1950 \\
\hline Europe $\ldots \ldots \ldots \ldots \ldots \ldots$ & 1.067 & 1.090 & 1.105 \\
\hline A mérique du Nord et du Centre ... & 364 & 618 & 625 \\
\hline Amérique du Sud $\ldots \ldots \ldots \ldots \ldots$ & 119 & 163 & 162 \\
\hline Asie $\ldots \ldots \ldots \ldots \ldots \ldots \ldots$ & 22 & 23 & 23 \\
\hline Afrique $\ldots \ldots \ldots \ldots \ldots \ldots \ldots$ & 6 & 17 & 17 \\
\hline Océanie $\ldots \ldots \ldots \ldots \ldots \ldots$ & 120 & 145 & 153 \\
\hline & 1.698 & 2.056 & 2.085 \\
\hline
\end{tabular}

Amérique du Nord et du Centre (U.S.A.) ; 43.000 tonnes en Amérique du Sud (Argentine) ; 38.000 tonnes en Europe; 33.000 tonnes en Océanie; 11.000 tonnes en Afrique; et 1.000 tonnes en Asie.

Les augmentations pour cent, pour les productions respectives d'avant-guerre, ont été les suivantes : Afrique, $200 \%$; Amérique du Nord et du Centre, $71 \%$; Amérique du Sud, 36\%; Océanie, $27 \%$; Europe, $4 \%$ environ.

La contribution au développement de la production des fromages qui est restée stationnaire en Europe par suite de raisons évidentes subséquentes à la période de guerre, doit être attribuée aux pays suivants : Tchécoslovaquie, Danemark, Finlande, Pays-Bas, Norvège, Suède, Suisse et Royaume-Uni, comme il apparaît dans le tableau suivant :

\begin{tabular}{|c|c|c|c|}
\hline \multirow{2}{*}{ Pays } & \multicolumn{3}{|c|}{$\begin{array}{l}\text { Production de fromage } \\
\text { en milliers de tonnes }\end{array}$} \\
\hline & $1937-1939$ & 1949 & 1950 \\
\hline Albanie $\ldots \ldots \ldots \ldots \ldots \ldots$ & 3 & 3 & 3 \\
\hline Autriche $\ldots \ldots \ldots \ldots \ldots \ldots \ldots$ & 27 & 14 & 16 \\
\hline Belgique $\ldots \ldots \ldots \ldots \ldots \ldots$ & 7 & 6 & 8 \\
\hline Tchécoslovaquie ........... & 11 & 14 & 14 \\
\hline 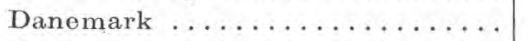 & 33 & 64 & 61 \\
\hline Finlande $\ldots \ldots \ldots \ldots \ldots \ldots$ & 11 & 12 & 14 \\
\hline France $\ldots \ldots \ldots \ldots \ldots \ldots \ldots$ & 270 & 240 & 250 \\
\hline Sarre $\ldots \ldots \ldots \ldots \ldots \ldots \ldots$ & 2 & 2 & 2 \\
\hline Allemagne Occidentale ......... & 135 & 149 & $=137$ \\
\hline Allemagne Orientale ........... & 7 & 7 & 7 \\
\hline Grèce $\ldots \ldots \ldots \ldots \ldots \ldots \ldots \ldots \ldots \ldots$ & 55 & 29 & 32 \\
\hline Hongrie $\ldots \ldots \ldots \ldots \ldots \ldots \ldots$ & 3 & 3 & 3 \\
\hline Irlande $\ldots \ldots \ldots \ldots \ldots \ldots \ldots$ & 3 , & 3 & 3 \\
\hline
\end{tabular}




\begin{tabular}{l|r|r|r} 
Italie (1) $\ldots \ldots \ldots \ldots \ldots \ldots \ldots \ldots \ldots$ & 237 & 237 & 238 \\
Pays-Bas $\ldots \ldots \ldots \ldots \ldots \ldots \ldots \ldots \ldots$ & 121 & 130 & 127 \\
Norvège $\ldots \ldots \ldots \ldots \ldots \ldots \ldots \ldots \ldots \ldots$ & 18 & 21 & 25 \\
Suède $\ldots \ldots \ldots \ldots \ldots \ldots \ldots \ldots \ldots$ & 34 & 66 & 52 \\
Suisse $\ldots \ldots \ldots \ldots \ldots \ldots \ldots \ldots \ldots \ldots$ & 51 & 54 & 56 \\
Royaume-Uni $\ldots \ldots \ldots \ldots \ldots \ldots \ldots$ & 39 & 36 & 57 \\
\hline & 1.067 & 1.090 & 1.105 \\
\hline
\end{tabular}

La production mondiale de lait condensé, qui était de 1.710 .000 tonnes avant la guerre et avait atteint en 1948 le chiffre de 2.530 .000 tonnes, pour redescendre à 2.405 .000 tonnes en 1949, est remontée en 1950 à 2.603.000 tonnes, ce qui correspond à une augmentation de $52 \%$ sur la production d'avant-guerre, (voir tableau ci-dessous) :

\begin{tabular}{|c|c|c|c|}
\hline \multirow[t]{2}{*}{ Pays } & \multicolumn{3}{|c|}{$\begin{array}{l}\text { Production de lait condensé } \\
\text { en milliers de tonnes }\end{array}$} \\
\hline & 1937.1939 & 1949 & 1950 \\
\hline Europe $\ldots \ldots \ldots \ldots \ldots \ldots \ldots \ldots$ & 426 & 391 & 528 \\
\hline A mérique du Nord et Centre ...... & 1.219 & 1.883 & 1.928 \\
\hline Amérique du Sud $\ldots \ldots \ldots \ldots \ldots$ & 21 & 23 & 27 \\
\hline 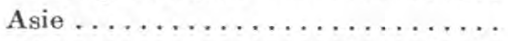 & - & - & - \\
\hline Afrique $\ldots \ldots \ldots \ldots \ldots \ldots \ldots \ldots \ldots$ & 13 & 14 & 17 \\
\hline Océanie $\ldots \ldots \ldots \ldots \ldots \ldots \ldots \ldots \ldots$ & 31 & 94 & 103 \\
\hline & 1.710 & 2.405 & 2.603 \\
\hline
\end{tabular}

Les augmentations en quantité et pour cent entre les chiffres d'avant-guerre et ceux de 1950 sont les suivantes : Amérique du Nord et du Centre, 709.000 tonnes (58\%); Europe, 102.000 tonnes $(24 \%)$; Océanie, 72.000 tonnes $(32 \%)$; Amérique du Sud, 6.000 tonnes $(30 \%)$; Afrique, 4.000 tonnes, $(30 \%)$.

L'augmentation la plus sensible constatée dans les productions de l'Amérique du Nord, est sur le lait entier (523.000 tonnes) et le lait écrémé (186.000 tonnes).

Les variations ascendantes intéressant l'Europe sont attribuées aux pays suivants : Belgique, Danemark, France, Allemagne Occidentale, Pays-Bas, Norvège, comme il apparaît dans le tableau suivant :

(1) Le relevé de " l'Assolatte "indique les quantités suivantes :

Avant la guerre : 200.000 tonnes; en 1949:247.000 tonnes; en 1950:270.000 tonnes. 


\begin{tabular}{|c|c|c|c|}
\hline \multirow[t]{2}{*}{ Pays } & \multicolumn{3}{|c|}{$\begin{array}{l}\text { Production de lait condensé } \\
\text { en milliers de tonnes }\end{array}$} \\
\hline & $1937-1939$ & 1949 & 1950 \\
\hline Belgique $\ldots \ldots \ldots \ldots \ldots \ldots \ldots$ & 3 & 2 & 5 \\
\hline Tehécoslovaquie .............. & 2 & 2 & 2 \\
\hline Danemark $\ldots \ldots \ldots \ldots \ldots \ldots$ & 17 & 37 & 37 \\
\hline France $\ldots \ldots \ldots \ldots \ldots \ldots \ldots \ldots$ & 24 & 28 & 43 \\
\hline Allemagne Occidentale .......... & 32 & 71 & 85 \\
\hline Irlande $\ldots \ldots \ldots \ldots \ldots \ldots \ldots$ & 8 & 9 & 9 \\
\hline Pays-Bas $\ldots \ldots \ldots \ldots \ldots \ldots \ldots$ & 143 & 118 & 173 \\
\hline Norvège $\ldots . . . \ldots \ldots \ldots \ldots . . . . .$. & 2 & 7 & 9 \\
\hline 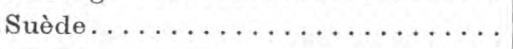 & 2 & 3 & 1 \\
\hline Suisse $\ldots \ldots \ldots \ldots \ldots \ldots \ldots$ & 6 & 6 & 4 \\
\hline \multirow[t]{2}{*}{ Royaume-Uni $\ldots \ldots \ldots \ldots \ldots$} & 187 & 108 & 160 \\
\hline & 426 & 391 & 528 \\
\hline
\end{tabular}

Le lait en poudre, dont la fabrication était limitée à 244.000 tonnes en 1937-1939, a marqué en 1950 une augmentation de production de 442.000 tonnes, c'est-à-dire $200 \%$ de la production d'avant-guerre.

La répartition de cette fabrication dans les divers continents est exprimée par le tableau suivant :

\begin{tabular}{|c|c|c|c|}
\hline \multirow[t]{2}{*}{ Pays } & \multicolumn{3}{|c|}{$\begin{array}{l}\text { Production de lait en poudre } \\
\text { en milliers de tonnes }\end{array}$} \\
\hline & $1937-1939$ & 1949 & 1950 \\
\hline Europe $\ldots \ldots \ldots \ldots \ldots \ldots \ldots$ & 80 & 122 & 139 \\
\hline Amérique du Nord et Centre ...... & 131 & 526 & 497 \\
\hline Amérique du Sud $\ldots \ldots \ldots \ldots \ldots$ & 6 & 13 & 16 \\
\hline 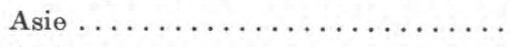 & - & - & - \\
\hline Afrique $\ldots \ldots \ldots \ldots \ldots \ldots \ldots$ & 1 & 1 & 1 \\
\hline \multirow[t]{2}{*}{ 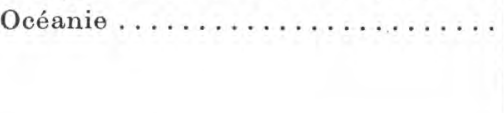 } & 26 & 71 & 83 \\
\hline & 244 & 733 & 736 \\
\hline
\end{tabular}

Les augmentations les plus sensibles de la production de lait en poudre ont été enregistrées : en Amérique du Nord, 366.000 tonnes de plus qu'avant-guerre (280\% d'augmentation); en Océanie, 57.000 tonnes $(220 \%)$; et en Europe, 59.000 tonnes $(70 \%)$. Dans les pays européens, la plus grande augmentation est cons- 
tatée au Danemark, en France, en Allemagne, en Suède, en Suisse et au Royaume-Uni, comme il apparaît dans le tableau suivant :

\begin{tabular}{|c|c|c|c|}
\hline \multirow[t]{2}{*}{ Pays } & \multicolumn{3}{|c|}{$\begin{array}{l}\text { Production de lait en poudre } \\
\text { en milliers de tonnes }\end{array}$} \\
\hline & $1937-1939$ & 1949 & 1950 \\
\hline Belgique ................ & 3 & 4 & 4 \\
\hline Tehécoslovaquie ........... & 1 & 1 & 1 \\
\hline 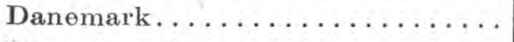 & 2 & 10 & 12 \\
\hline France $\ldots \ldots \ldots \ldots \ldots \ldots$ & 3 & 6 & 6 \\
\hline Allemagne Occidentale ......... & 9 & 21 & 17 \\
\hline Irlande $\ldots \ldots \ldots \ldots \ldots \ldots$ & 1 & 1 & 1 \\
\hline Pays-Bas $\ldots \ldots \ldots \ldots \ldots \ldots$ & 37 & 28 & 41 \\
\hline Norvège $\ldots \ldots \ldots \ldots \ldots \ldots \ldots$ & 2 & 1 & 1 \\
\hline Suède............... . . . . . & 1 & 15 & 9 \\
\hline Suisse $\ldots \ldots \ldots \ldots \ldots \ldots \ldots$ & +3 & 6 & 6 \\
\hline \multirow[t]{2}{*}{ Royaume-Uni . } & 18 & 29 & 41 \\
\hline & 80 & 122 & 139 \\
\hline
\end{tabular}

La régression de la fabrication du lait entier en poudre, par rapport à l'augmentation de la production de lait ecrémé en poudre, déjà relevée en 1949, s'est maintenue stationnaire aux U.S.A. et au Canada, si bien que la production des dits pays en 1950 se répartit ainsi : 65.000 tonnes pour le lait entier, et 432.000 tonnes pour le lait écrémé.

La même direction a été suivie en Europe également, par les Pays-Bas, qui ont produit 14.000 tonnes de lait entier contre 27.000 tonnes de lait écrémé en poudre, alors que le Danemark, la Suède et la Suisse ont produit au total 16.000 tonnes de lait entier et 11.000 de lait écrémé.

\section{BIBLIOGRAPHIE ANALYTIQUE}

\section{LES LIVRES}

Braconnier (R.), Glandard (J.) et Collaborateurs. - Nouveau Larousse Agricole. 1.230 pages, format in-4 ${ }^{\circ}$, 56 hors texte, en couleur et en noir, $23 \mathrm{pl}$. en noir, 1.335 gravures dans le texte. Editeur : Larousse, 13-31, rue Montparnasse et 114, boul. Raspail $\left(6^{\mathrm{e}}\right)$, Paris, 1952. Prix : chaque fascicule, 280 francs; relié, 5.900 francs. 